\title{
Cognitive impairment in patients with epilepsy: A case control study at a tertiary level hospital in India
}

\author{
* Dr.Ravindra B. Narod ${ }^{1}$, Dr.Rathod Rahul Narayanrao ${ }^{2}$, Dr.Sayali V. Bhambar ${ }^{3}$ \\ Dr.Sapna Anjutagi ${ }^{4}$, Dr.Smit U. Janrao ${ }^{2}$, Dr.Pranav Deore ${ }^{5}$ \\ ${ }^{1}$ Assistant Professor, Department of Psychiatry Government Medical College, Miraj, Maharashtra \\ ${ }^{2}$ Assistant Professor, Department of Medicine Byramjee Jeejeebhoy Government Medical College \\ And Sassoon General Hospitals, Pune \\ ${ }^{3}$ Resident, Department of Chest Medicine MVP's Dr. Vasantrao Pawar Medical College, Nashik \\ ${ }^{4}$ Assistant Professor, Department of Pediatrics Bharati Vidyapeeth Deemed \\ University Medical College, Pune \\ ${ }^{5}$ Resident, Department of Medicine Dr. Vasantrao Pawar Medical College, Nashik \\ Corresponding author: * Dr.Ravindra B. Narod
}

\begin{abstract}
:
Introduction: Cognition is defined as the capacity of the brain to process information accurately leading to an adaptive behaviour. This study aims at understanding the decline in cognition in patients diagnosed with epilepsy in India using a standardized cognition scale.

Methodology: We did a case control study in the Department of Psychiatry, Government Medical College, Miraj in which all patients with a diagnosis of epilepsy from October 2016 till February 2017 were included. Patients were tested for cognitive impairment using the PGI Memory scale (PGIMS). Controls were included from the population sample of non teaching staff of college. We noted age, gender, handedness, and education levels and clinical information. All cases and controls were tested for cognitive functioning using PGIMS. The data was analysed descriptively and then the cases and controls were compared using the unpaired Student's $t$ test, with $p$ value less than 0.05 being statistically significant.

Results: We enrolled 60 patients diagnosed with epilepsy and same number of healthy controls from the same insitution. Average age of the patients with epilepsy was $43.7 \pm 2.4$ years and that of controls was $48.1 \pm 3.6$ years. The difference between the cases and controls was found to be statistically highly significant in cognition tests like recent memory, remote memory, immediate recall and visual retention. On cognitive tests like attention and concentration, retention of dissimilar pairs, mental balance the difference between the groups was found to be statistically significant.

Conclusions: Findings of our study demonstrates that patients with epilepsy shuould be routinely tested for cognitive decline.
\end{abstract}

Keywords: cognition, epilepsy, epidemiology, memory, intelligence

\section{Introduction}

A seizure is a sudden change in behavior caused by electrical hypersynchronization of neuronal networks in the cerebral cortex. Seizures are a common occurrence, affecting an estimated 8 to $10 \%$ of the population over a lifetime. ${ }^{1}$ Epilepsy is defined when at least two unprovoked (or reflex) seizures occur more than 24 hours apart, or one unprovoked (or reflex) seizure and a probability of further seizures similar to the general recurrence risk after two unprovoked seizures, occurring over the next 10 years or has a diagnosis of an epilepsy syndrome. ${ }^{2}$ Cognition is defined as the capacity of the brain to process information accurately leading to an adaptive behaviour. It involves numerous higher functions of the brain like ability to solve routine problems, to memorize information, and to focus and concentrate. ${ }^{3}$ Previous studies have demonstrated that epilepsy can result in decline in cognitive function of the patient. ${ }^{4,5}$ There are numerous patient related factors which can play a role in declining cognitionof the patient. The type of clinical epilepsy syndrome, areas of the brain involved, treatment given etc, all can affect the level of cognition of the patient. There is scarcity of data which has studied the effect of epilepsy on cognition of the patient. This study aims at understanding the decline in cognition in patients diagnosed with epilepsy in India using a standardized cognition scale.

\section{Study Design and sample population}

\section{Methodology}

We designed a case control study in the Department of Psychiatry, Government Medical College, Miraj. We considered all patients who presented in the outpatient clinic and were diagnosed with epilepsy from 
October 2016 till February 2017. Some of the diagnosed patients were referred for cognitive testing to the Department of Psychiatry. We used Kolkata Cognitive Screening battery ${ }^{6}$ for screening the patients and then tested for cognitive impairment using the PGI Memory scale (PGIMS) ${ }^{7}$. PGIMS is a standardized and validated Indian tool for evaluating the cognitive and memory ability in patients. Our inclusion criteria for the study was all patients with temporal or extra temporal epilepsy, aged between 18 to 60 years, having diagnosed with epilepsy for atleast 6 months, of both genders, all education levels, urban as well as rural backgrounds, right or left handed and those who consented to participate in the study. We excluded patients below 18 years or more than 60 years of age, diagnosed with mental retardation, had history of psychiatric illness, head injury, systemic illness or refused to consent for the study. The diagnosis of epilepsy was made according to the definition given by the International League Against Epilepsy. ${ }^{8}$ These patients served as cases in this study. Age and gender matched controls were selected and included in the study as well. Controls were included from the population sample of non teaching staff of Government Medical College, Miraj. After approval of the institutional ethics committee the study subjects were approached for enrollment if they fulfilled the inclusion and exclusion criteria. The aim and process of the study methodology was explained to the subjects. Informed written consent was obtained from the subjects.

\section{Data Collection and Data Analysis}

A pretested semistructured questionnaire was designed and administered to all the subjects enrolled in the study. We collected socio-demographic information of each of the subject and compared the two groups to ensure comparability. This included age, gender, handedness, and education levels. Then we collected clinical information of the subjects. All patients with a diagnosis of epilepsy who underwent screening using a Kolkata Cognitive Screening Battery were administered PGIMS. The findings on the scale was noted. Similarly, controls were administered the PGIMS as well and their readings were noted. The data were entered in excel sheets to prepare the master chart and imported in SPSS version 17. The data was analysed descriptively and then the cases and controls were compared using the unpaired Student's $t$ test, with $p$ value less than 0.05 being statistically significant.

\section{Results}

We enrolled 60 patients diagnosed with epilepsy and same number of healthy controls from the same insitution. Both of these groups were age and gender matched (Table 1). Average age of the patients with epilepsy was $43.7 \pm 2.4$ years and that of controls was $48.1 \pm 3.6$ years. The subjects in both the groups were similar with respect to their handedness and education level as well. The groups were compared using an unpaired $t$ test to check for statistical difference in their cognition. The difference between the cases and controls was found to be statistically highly significant ( $\mathrm{p}$ value less than 0.001 ) in cognition tests like recent memory, remote memory, immediate recall and visual retention (Table 2). On cognitive tests like attention and concentration, retention of dissimilar pairs, mental balance the difference between the groups was found to be statistically significant ( $\mathrm{p}$ value less than 0.01 ). The difference in delayed recall and recognition was reported to be statistically insignificant between epileptics and healthy controls.

\section{Discussion}

Our study has demonstrated that there is a significant decline in the cognition of patients with epilepsy. Using a validated standardized cognition scale, epilepsy patients in our study have reported statistically significantly lower scale scores as compared to controls in the study. Capacity of brain such as intelligent thinking, problem solving, perceiving, expressing, remembering remotely and recently, reasoning, and understanding are included in cognition. Cognition has a very important role in the creation, evolution, and divulging of many of these aspects of higher brain function and studies have reported these functions to be compromised in people with epilepsy. Onset of most cases of epilepsy is in childhood, and thus the start of seizure activity coincides with that phase of brain development when it most sensitive to such insults. It has been reported that the majority of patients with epilepsy have normal intelligence, however the distribution of Intelligent Quotient (IQ) scores is seen to be towards lower values. ${ }^{9}$ Smith et al demonstrated that 51 children with medically refractory epilepsy had wide range of cognitive functioning. ${ }^{10}$

A number of factors related to the patient influence the neuropsychiatric effect of epilepsy, such as cause of epilepsy, location of epileptogenic focus in the brain, age at onset and duration of epilepsy syndrome, the type of the epilepsy syndrome, medications taken by the patient, and other social factors. Studies have reported that epilepsies that develop as a result of brain trauma are more associated with cognitive and behavioral changes due to brain injury. ${ }^{11}$ The site of an epileptogenic focus is also important in determining how severe the cognitive deficits will there be. As it has been seen that moe of memory deficits may be seen with temporal lobe epilepsy, and executive functioning may be disturbed with frontal lobe epilepsy. ${ }^{12}$ Complex partial seqizures are most likely to be related to behavioral disturbances. 
Similarly, lower intelligence quotient (IQ) has been linked to onset of epilepsy before 5 years of age.In late onset epilepsy behavioral symptoms are more prominent. Generalized tonic-clonic seizures as compared to partial seizures result in more cognitive impairment than that observed. ${ }^{13}$

One of the most common complaints of patients with epilepsy are memory problems. A survery by Thompson and Corcoran showed that people with epilepsy had higher frequency of memory failures and tendency to forget words. ${ }^{14}$ Previously published studies have also reported that anticonvulsants are associated with insomnia, altered sleep patterns and in a few cases with worsening of breathing during sleep. All these may have an adverse effect on cognition of the patient. Some of the limitations of our study includes small sample size and single center study. Our results may not generalizable to other geographical areas and therefore future studies should aim for multi-centric studies.

\section{Conclusion}

Our study shows that patients with epilepsy have severe cognitive decline and therefore all patients with epilepsy should be screened for cognitive impairment using standardized memory scales. The treatment plans for such patients should be customised keeping theese associated morbidities in mind.

Table 1: Baseline demographic characteristics of patients enrolled in the study

\begin{tabular}{|llll|}
\hline Number of subjects & Cases & Controls & p value \\
Males & 60 & 60 & 0.26 \\
Average age & 38 & 32 & 0.09 \\
Right handed & $43.7 \pm 2.4$ years & $48.1 \pm 3.6$ years & 0.47 \\
Education level of the patients & 43 & 46 & 0.83 \\
Illiterate & 2 & & \\
Primary school & 8 & 1 & \\
Middle school & 15 & 10 & \\
High school & 14 & 14 & \\
Intermediate school & 18 & 18 & \\
Graduate or higher & 3 & 1 & \\
\hline
\end{tabular}

Table 2: Comparison of cases and controls on PGI Memory scale

\begin{tabular}{|llll|}
\hline Cognitive test & $\begin{array}{l}\text { Cases } \\
(\text { Mean } \pm \text { SD*) }\end{array}$ & $\begin{array}{l}\text { Controls } \\
(\text { Mean } \pm \text { SD*) }\end{array}$ & p value** \\
Recent memory & $4.28 \pm 1.53$ & $5.21 \pm 0.24$ & $<0.001$ \\
Remote memory & $4.34 \pm 0.23$ & $5.38 \pm 0.47$ & $<0.001$ \\
Attention \& concentration & $3.82 \pm 0.89$ & $4.72 \pm 0.11$ & $<0.01$ \\
Immediate recall & $7.23 \pm 1.82$ & $8.15 \pm 0.28$ & $<0.001$ \\
Delayed recall & $5.21 \pm 0.68$ & $5.84 \pm 1.04$ & 0.48 \\
Retention for similar pairs & $6.23 \pm 1.71$ & $7.29 \pm 1.45$ & 0.092 \\
Retention for dissimilar pairs & $3.14 \pm 0.17$ & $4.67 \pm 0.53$ & $<0.01$ \\
Mental balance & $8.11 \pm 1.32$ & $9.46 \pm 0.23$ & $<0.01$ \\
Visual retention & $7.81 \pm 2.92$ & $9.57 \pm 0.43$ & $<0.001$ \\
Recognition & $7.19 \pm 1.34$ & $8.12 \pm 1.72$ & 0.66 \\
\hline
\end{tabular}

$* \mathrm{SD}=$ standard deviation

**p value calculated from unpaired t test

\section{References}

[1]. Annegers JF, Hauser WA, Lee JR, Rocca WA. Incidence of acute symptomatic seizures in Rochester, Minnesota, 1935-1984. Epilepsia 1995; 36:327.

[2]. Fisher RS, Acevedo C, Arzimanoglou A, et al. ILAE official report: a practical clinical definition of epilepsy. Epilepsia 2014; 55:475.

[3]. Petersen RC, Smith G, Kokmen E, et al. Memory function in normal aging. Neurology 1992; 42:396.

[4]. Oostrom KJ, van Teeseling H, Smeets-Schouten A, Peters ACB, Jennekens-Schinkel A. Three to four years after diagnosis: cognition and behaviour in children with 'epilepsy only'. A prospective, controlled study. Neurology 2005;128:1546- 55 .

[5]. Austin JK, Dunn DW, Johnson CS, Perkins SM. Behavioral issues involving children and adolescents with epilepsy and the impact of their families: recent research data. Epilepsy \& Behavior. 2004 Oct 31;5:33-41.

[6]. Das SK, Banerjee TK, Mukherjee CS, Bose P, Biswas A, Hazra A, et al. An urban community-based study of cognitive function among non-demented elderly population in India. Neurol Asia. 2006;11:37-48

[7]. Pershad D, Wig NN. A battery of simple tests of memory for use in India. Neurology India. 1976 Jun;24(2):86. 
[8]. Trinka E, Cock H, Hesdorffer D, Rossetti AO, Scheffer IE, Shinnar S, Shorvon S, Lowenstein DH. A definition and classification of status epilepticus-Report of the ILAE Task Force on Classification of Status Epilepticus. Epilepsia. 2015 Oct 1;56(10):1515-23.

[9]. Sillanpaa M. (1990) Children with epilepsy as adults: Outcome after 30 years of follow-up. Acta Paediatr Scand Suppl 368: $1-78$.

[10]. Smith ML, Elliott IM, Lach L. (2002) Cognitive skills in children with intractable epilepsy: Comparison of surgical and non-surgical candidates. Epilepsia 43: $631-637$.

[11]. Silver JM, McAllister TW, Arciniegas DB. Depression and cognitive complaints following mild traumatic brain injury. American Journal of Psychiatry. 2009 Jun;166(6):653-61.

[12]. Petersen RC, Negash S. Mild cognitive impairment: an overview. CNS spectrums. 2008 Jan;13(1):45-53.

[13]. Mesulam, 1998. Mesulam M.M.: From sensation to cognition. Brain 1998; 121: pp. 1013-1052

[14]. Thompson PJ, Corcoran 1R. (1992) Everyday memory failures in people with epilepsy. Epilepsia 33 (Suppl 6): S18 20.

Dr.Ravindra B. Narod. "Cognitive impairment in patients with epilepsy: A case control study at a tertiary level hospital in India." IOSR Journal of Dental and Medical Sciences (IOSR-JDMS) 16.7 (2017): 103-06. 